\title{
群组协作社交网络关系的分析及可视化
}

\author{
路强 ${ }^{1,2,3)}$, 黄静 2 ), 盛开维 ${ }^{2}$, 徐文强 2 ) \\ ${ }^{1)}$ (大数据知识工程教育部重点实验室(合肥工业大学) 合肥 230009) \\ 2) (合肥工业大学计算机与信息学院 合肥 230601) \\ 3) (智能互联系统安徽省实验室(合肥工业大学) 合肥 230009) \\ (luqiang@hfut.edu.cn)
}

\begin{abstract}
摘 要: 社交网络的研究应用领域广泛, 大多数的研究主要关注于社交网络结构中节点和链接的变化, 研究角度较 为单一. 而群组协作关系在社交网络中较为普遍, 为更好地探索群组协作过程中群组级别网络结构的演变, 首先将 组级任务添加到用于图可视化的任务分类中, 针对协作关系网络的特性, 设置一个适用于群组协作关系分析的任务 分类法, 根据此任务分类法设置探究群组协作需完成的任务; 然后设计相关可视化视图, 以更直观的方式展示群组 之间以及群组内部协作关系的特征以及随时间推进的演变模式; 最后在学术协作文献数据集上进行具体案例分析, 通过用户评估实验，采用李克特量表对调查问卷进行分析，验证了设计可视化视图的有效性和实用性，可为社交网 络关系分析人员提供群组协作的可视化分析方法, 更便于对群组协作网络关系的进一步探究和应用.
\end{abstract}

关键词：社交网络; 群组协作; 可视化分析; 任务分类; 可视化设计

中图法分类号: TP391.41

DOI: $10.3724 /$ SP.J.1089.2021.18430

\section{Analysis and Visualization of Group Collaborative on Social Networks Relationships}

\author{
Lu Qiang ${ }^{1,2,3)}$, Huang Jing ${ }^{2)}$, Sheng Kaiwei ${ }^{2)}$, and Xu Wenqiang ${ }^{2)}$ \\ 1) (Key Laboratory of Knowledge Engineering with Big Data (Hefei University of Technology), Hefei 230009) \\ 2) (School of Computer and Information, Hefei University of Technology, Hefei 230601) \\ 3) (Intelligent Interconnected Systems Laboratory of Anhui Province (Hefei University of Technology), Hefei 230009)
}

\begin{abstract}
The study of social networks is widely applied, which is mainly focused on the change of the social network structure of node and link, and the research angle is relatively single. Group collaboration is common in social networks. To better explore the evolution of group-level network structure in the process of group collaboration, this paper first adds group-level tasks to the task classification for graphic visualization. A task taxonomy suitable for group collaboration relationship analysis is set up according to the characteristics of collaborative relationship networks and tasks to be completed for group collaboration are set up according to this task taxonomy. Then, relevant visual views are designed to display the features and evolution patterns of collaborative relationships among and within groups in a more intuitive way. Finally, a specific case analysis was carried out on the academic collaboration literature data set. Through user evaluation experiments, the Likert scale was used to analyze the questionnaire to verify the effectiveness and practicability of the design visualization view, so as to provide a visual analysis method of group collaboration for social network relationship analysts, facilitating further exploration and application of group collaboration network relationships.
\end{abstract}

收稿日期: 2020-06-09; 修回日期：2020-11-28. 基金项目：国家自然科学基金(61972130); 安徽省黄山市科技计划(2019KN-05). 路强(1978一), 男, 博士, 副教授, 硕士生导师, CCF 会员, 主要研究方向为可视化与可视分析、计算机图形学; 黄静(1995一), 女, 硕士 研究生, 主要研究方向为可视化与可视分析; 盛开维(1995-), 男, 硕士研究生, 主要研究方向为可视化与可视分析; 徐文强(1997一), 男, 硕士研究生, 主要研究方向为可视化与可视分析. 
Key words: social network; group collaborative; visual analysis; task classification; visual design

社交网络越来越多地确立为在个人之间建立 和加强社交关系的主要手段之一 ${ }^{[1]}$. 尽管人类学文 献已经获得了描述人类社交网络的属性的实质性 结果, 但仍需要对社交网络的属性有一个清晰的 了解. 如今, 社交网络分析已成为研究社会中信息 流和人际关系的重要方法. 社会学家、心理学家和 人类学家主要关注社交网络的 2 个方面：对大量人 的关系进行量化的社会中心分析, 以及研究与自 我相关联的人们和特定个体之间的动态关系的自 我中心分析.

可视化是一种创建图像、图表或动画以传达消 息的技术，是直观地表达抽象信息的有效方法. 社 交网络可视化是指构建社会行动者之间联系的图 形化图像的实践. 使用可视化视图展示动态社交 网络关系的演化, 可以让人们观察社交网络关系 的演化模式, 以预测将来事态的发展趋势. 如对学 术协作网络的分析可以看出感兴趣作者的合作作 者和机构及研究领域的演化模式, 以分析其在未 来更有可能与哪个机构合作, 更倾向于在哪个领 域做学术研究工作; 通过分析和可视化一个企业 的社交网络关系，可以帮助企业更好地制定未来 发展计划, 制定更精准、广泛和有效的社会化营销 体系，从而提高服务认知，提升市场销售.

组织和分析大型数据集合通常涉及以可视化 方式揭示数据元素的属性和关系. 在许多应用领 域, 如社会科学(社交网络)、生物学(蛋白质相互作 用模型)、软件工程(程序调用图)中连通性和拓扑信 息与分组和集群信息交织在一起. 例如, 生物学家 试图了解人类基因组中基因组之间的关系, 社会 科学家通过识别社交网络中的社区来研究人与人 之间的互动, 而机器学习专家则试图了解他们的 数据是如何分类的. 通常, 使用可视化工具可以帮 助更好地汶览大型数据集的数据. 群组的视觉表 示形式很多，每种形式都会影响人们如何感知元 素的属性以及与周围元素的关系. 图和网络可视 化的任务分类主要关注在分析图的连接性和拓扑 结构时经常遇到的任务.

最近出现的一些可视化技术，都明确地使用 了分组和集群, 但由于缺乏标准化的组级任务, 因 此很难对这些可视化效果进行评估. 考虑到这一 点, 本文的目标是定义一组新的任务来评估对组 级的理解. 为此, 提出了几种组级任务类型, 并提 供了每种类型的几个示例. 使用抽象可视化任务
的多层次类型来描述一些被提议的任务. 将组级 任务添加到用于图可视化的任务分类中, 将使该 分类对最近的图可视化技术更加有用. 它将帮助 评估人员定义和分类新的任务, 将有助于归纳在 受控实验中收集到的结果并进行分析. 然后, 根据 提出的组级任务设计可视化视图, 动态显示不同 时期群组之间关系的演变, 以探索群组合作的规 律以及预测未来变化趋势. 最后, 通过用户评估验 证本文提出的组级任务分类以及可视化视图的实 用性和有效性.

\section{1 相关工作}

\section{1 动态网络关系可视化}

研究者已经提出了许多可视化技术来探索和 分析网络结构的动态演进模式. 动态网络的分析 和可视化方法主要分为动画法和时间线法, 以及 两者的综合使用 ${ }^{[2-3]}$.

动画法是通过在时间而非在空间中多路复用动 态网络的状态来降低网络复杂性的有效手段. Eades 等 ${ }^{[4]}$ 首先使用动画法来方便用户汶览自己感兴趣的 图表, 实现视图的平滑转换. GraphDiaries ${ }^{[5]}$ 依赖于 动画过渡的交互技术，突出显示时间步之间网络 结构的变化, 方便用户识别和理解这些变化. 动画 法在展示网络演化和过渡过程上展现了很大的优 势 ${ }^{[6-7]}$, 但是, 当网络规模很大时, 视图的转换往 往会造成视觉的混乱, 反而不利于观察到一些变 化的细节 ${ }^{[8]}$.

时间线法可以在一张静态图片中展现一段时 间内的网络结构变化, 用户通过比较不同时间步 长的网络快照来监测网络变化. $\mathrm{He}$ 等 $^{[9]}$ 利用时间轴 的推移来展示共同作者网络的演变, 帮助用户找 到合作作者相关信息的变化, 如合作的作者数量 和论文领域等. Ogawa 等 ${ }^{[10]}$ 提出了 Storyline 的可视 化方法来表现软件开发过程中人员之间的协作关 系, 许多研究者改进了其布局算法, 并将其应用于 网络结构的演变可视化领域 ${ }^{[1-13]}$.

一些研究者也使用动画法和时间线的混合技 术来可视化网络结构. Beck 等 $^{[14]}$ 介绍了一种基于 Parallel Edge Splatting 可变时间图的可视化技术, 将图形序列动画化为移动时间线表示; 文献 [15-16]使用基于时间轴的聚合演化表示集群演化 来汶览动画节点链接图, DiffAni ${ }^{[6]}$ 允许用户以交互 
方式将时间线表示的各部分聚合成动画. 这些可 视化方法可以很好地展示随时间演变整体网络的 演化模式，但是无法很好地描述特定的群组信息.

\section{2 群组协作关系可视化}

针对不受时空局限的、更为自然、便捷的协作 方式的特性，张立生 ${ }^{[17]}$ 提出了适用于移动协同领 域的研究模型, 从群组组织结构和群组协作过程 2 个方面深人探讨了与移动协同群组协作性能的 关系.

目前对于群组协作的可视化形式大多是关于 集合可视化的形式, 集合可视化的一种常见方法 是在空间上排列集合成员，以使每个集合形成一 个空间簇．当执行社交网络分析时，该方法在社区 检测中特别流行 ${ }^{[18]}$. 这些社区通常通过覆盖凸包 来表示 ${ }^{[19]}$, 并且通常作为社交网络上的附加层绘 制. 对于分散的数据集，使用凸包集的表示形式可 能会出现问题. 特别是在地图上表示特定兴趣点, 如一些边缘节点时，可能会误解集合的真实支撑区 域. 由于它覆盖的区域比真实区域大，如集合包含 位于地图 4 个角上的 4 个元素, 覆盖整个地图的区 域，因此错误地与该地图上的所有可能集合相交.

集合可视化可以很好地展示群组协作中的各 个群组的划分, 但是在协作模式的探究中缺乏更 直观的可视化形式, 因此本文重点探究群组协作 社交网络中群组之间以及群组内部个体之间的关 系演化可视化方法的实现, 并探索随时间推进网 络协作的演变规律.

\section{3 用于图可视化的任务分类}

图用于描述一组实体(节点)及其关系(链接). 图形网络通常使用节点链接关系图来表示, 其中 节点被描述为点, 链接被描述为连接相应点的线 段. 虽然任务分类可以用于广泛的可视化技术，包 括节点链接图，但是没有一个可视化技术是专门 处理带有附加分组信息图形的可视化的.

Brehmer 等 ${ }^{[20]}$ 组织了大量关于任务分类的可 视化工作, 突出了它们的优点和缺点. 他们指出, 大多数方法的主要缺点是缺乏任务的全局视图: 高层次的类别经常忽略如何执行任务, 而低层次 的类别经常忽略为什么执行任务. 为了弥补这一 缺陷，他们开发了一个多层次的任务分类，帮助创 建一个完整的任务描述. 这种多层次的类型包括 3 个问题: why, how 和 what. 然而, Brehmer 等 ${ }^{[20]}$ 的工 作并不是要取代面向模型的分类法, 而是要包含 并补充这些特定的分类系统. 实际上, 特定主题的 分类法提供了关于底层任务的详细信息, 这些底
层任务必须用 Brehmer 等 ${ }^{[20]}$ 非常通用的方法进行 抽象. 相反, 它们提供了将这些低级任务放在上下 文中的工具，指导评估设计人员提供诸如用户专 业知识和动机之类的信息.

Lee 等 ${ }^{[21]}$ 提出了一些他们认为在分析图形数 据时经常遇到的任务列表, 定义了特定于图形的 对象, 并演示如何将所有复杂任务视为在这些对 象上执行的一系列低级任务; 最终结论显示，与基 准数据集和特定任务相关联的分类法将有助于评 估人员总结通过一系列受控实验收集的结果.

\section{2 群组协作网络任务分类}

许多组级任务可以推断为与图形相关的任务 的扩展. 元数据是描述数据的数据, 是对数据及信 息资源的描述性信息. 许多节点组成的组可以表 达为是对这些节点的描述性信息，因此，可以将这 些组视为元数据节点, 根据原始节点之间是否存 在链接来定义元数据链接, 并在这个过程生成的 元数据图上执行图任务. 许多图的特征, 如度、邻 接或节点的中心性可直接转化为相关的组属性. 可以用几种不同的方式构建这个元数据图, 从而 对数据产生不同的见解. 例如, 在组共享其边界的 表示中, 可以基于组联系人构建可视化视图. 这种 可视化方式在某种意义上来说有效的, 如确定组 之间是否有更强的相互联系.

本节详细定义了多个组级任务的信息, 这些 任务来自使用诸如 LineSets ${ }^{[22]}$, GMap ${ }^{[23]}$ 和 Bubble

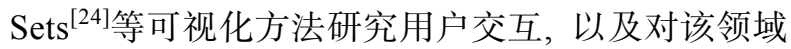
专家的访谈. 邀请可视化领域的专家学者, 请他们 列出使用上述 3 种可视化方法所遇到的组级分类 相关的问题，根据解决这些问题所需要的信息将 其分成 3 大类, 定义一组新的任务来评估群组级别 可视化效果.

（1）群组节点任务(T1). 仅考虑组和节点信息 即可执行此类别的任务. 例如, 查找节点数量最大 的组.

(2) 群组链接任务(T2). 仅考虑组和链接信息 即可执行此类别中的任务. 例如, 查找给定组中的 链接数.

(3) 群组网络任务(T3). 仅考虑组、节点和链 接信息即可执行该类任务. 例如, 查找联系最紧密 的群组.

下面详细地定义和分析每一项任务：

群组节点任务 (T1). 列出包含具有特定节点的 
组(如包含节点 $X$ 的组), 以及给定组的节点数. 查 找节点数最大最小的组; 给定 2 个节点 $X$ 和 $Y$, 检 查它们是否属于同一组; 给定一个节点 $V$ 具有特 定的特征, 找出与 $V$ 有链接关系的组.

群组链接任务(T2). 查找给定组中的链接数. 查找链接数量最大(最小)的组, 列出包含具有特定 特征的链接的组(如包含联系最紧密的链接); 查找 在组外连接该组节点的链接数最小 (最大)的组.

群组网络任务(T3). 查看群组之间的链接数, 即查看群组之间合作情况. 找到联系最稀疏(最密 集)的群体, 查看 $X$ 与 $Y$ 组节点之间的链接数目, 找出数目最多(最少)的一组; 找到路径 $U-W$, 节 点 $U$ 和 $W$ 在同一组吗? 给定 2 个不同组 $X$ 和 $Y$ 中 的节点, 查看其合作情况.

\section{3 可视化设计}

通过对群组协作网络的任务分析，本文提出 了一组新的组级任务分类. 针对上述任务分类, 对 需要可视化的群组元素进行可视化的元素映射, 设计相关的可视化视图, 以实现群组协作网络关 系的视图表达，协助探索群组协作网络的结构特
点以及随时间的演变规律. 节点链接图是信息可 视化中表现图结构数据最基本的方法, 本文可视 化视图设计在节点链接图上叠加群组信息, 设计 可视化元素对群组信息进行编码. 为了辅助用户 了解可视化视图中元素含义以及探索视图, 在可 视化视图的基础上添加一些交互手段, 可帮助用 户更加快速有效地汶览视图, 查看群组协作关系网 络的结构特征及其演化模式; 同时添加辅助分析视 图, 统计分析群组协作中群组内部节点以及链接的 变化情况. 下面将针对可视化视图的元素映射、交 互手段及辅助分析视图进行逐一介绍.

\section{1 元素映射}

数据可视化是数据到视觉元素的映射过程, 映射也是视觉编码的过程, 视觉元素也可称为视 觉通道. 使用可视化的方法描述群组关系时, 首先 要对群组数据中的基本元素和基本关系进行可视编 码, 即将基本元素和关系映射为可视化视图中的图 形元素. 此外, 理解基本图形元素的表达含义是理 解可视化视图, 从而进行快速分析的基础. 表 1 描 述了第 2 节提出的群组协作中的基本元素与可视化 视图中的图形元素之间的映射关系, 包括节点、群 组、链接的图形表示以及映射关系的详细描述.

表 1 群组元素和可视化视图中图形元素的映射关系

\begin{tabular}{lll}
\hline 元素 & 视觉变量映射 & \multicolumn{1}{c}{ 描述 } \\
\hline 节点 & 带颜色圆球 & 圆球颜色表示所属群组分组, 圆球大小表示该节 \\
点与其他节点合作权重, 权重越大, 圆球越大
\end{tabular}

\section{2 交互手段}

通过设计可视化元素映射和布局算法的实现， 群组以及群组之间协作关系的基本可视化视图可 以展示出来. 为了便于用户理解可视化视图中的 元素, 针对第 2 节中提到的群组组级任务分类, 在 基本的可视化视图上添加标签描述, 设置视图的 交互手段, 方便用户针对上述提出的任务对视图 进行探索. 本文的交互手段如下:

(1) 悬停标签. 为群组节点以及节点之间的链 接添加悬停标签进行辅助说明, 包括显示群组节 点的名称以及详细描述等和节点之间链接的详细
信息, 帮助用户进一步了解节点元素和链接元素 的具体含义. 具体方法如下: 当鼠标悬停在某个节 点上, 在节点旁边显示标签, 标签内容为节点所代 表的元素的具体名称; 当鼠标悬停在某条链接时, 在链接的上方显示标签, 标签内容为该条链接的 2 个节点的信息.

(2) 时间段选择. 为探索随时间推进的群组协 作关系的演变模式, 增加时间线的可视化辅助方 法, 帮助展示不同时间段的群组网络结构. 具体方 法如下: 添加下拉框, 可以选择任意时间段, 点击后 则可以显示该时间段对应的群组节点和节点之间的 
链接，用户可直观地分析该时间段的群组协作网络 的详细信息. 通过查看不同时间段的视图, 可以直 观地分析随时间推进群组内部以及群组之间节点元 素和链接元素的演变规律, 以推测未来的演变模式.

(3) 选择过滤. 当用户想要探究某个群组内部 的协作关系时，使用选择过滤和突出的方式突显 关键信息，使用户可以快速定位，减少搜索查看的 时间. 也可以选择多个特定群组, 查看这些群组之 间的协作关系. 具体方法如下：用户点击想要查看 的群组按钮, 视图将会突出显示该群组内部的节 点之间的链接情况，除被选择群组外其他群组的 节点和链接信息将被隐去.

除了上述交互手段外，为了适应更多用户的喜 好和适应多种应用场景，本文提供多种配色方法供 用户选择，即配色方案可调整. 如在多个群组协作 网络中, 某些群组之间合作情况较为紧密, 想要着 重分析这些群组之间的合作概况，可调整这些群组 代表颜色的对比度，使之对比更加鲜明，更加清晰 地展示相关链接信息, 方便用户进行深人分析.

\section{3 辅助分析视图}

基本的可视化视图可直观地展示群组之间以 及群组内部的协作关系. 考虑用户在探索视图时 需要了解一些统计信息, 本文添加辅助分析视图, 可帮助用户查看群组数目以及群组内部人员信息.

(1) 群组人员信息统计视图. 显示该群组内部 人员信息情况，如图 1 所示，用户如若对某一群组感 兴趣，可通过点击该群组对应的按钮，详细查看对 应时间段内该群组内有合作情况的所有成员信息.

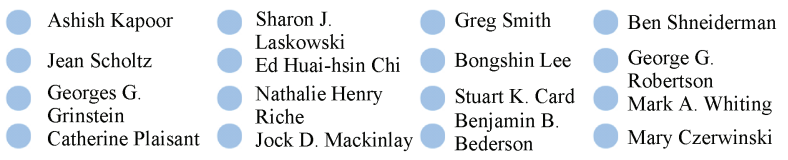

图 1 群组人员信息统计视图

(2) 群组内部合作信息统计视图. 展示某一时 间段内所有群组内部合作数目统计信息, 并添加悬 停标签显示特定群组的合作数目, 如图 2 所示.

\section{4 结果分析}

\section{1 案 例}

(1) 数据概述. 采用学术协作网络数据集 ${ }^{[25]}$ 验证本文提出的可视化视图. 从 IEEE VIS 会议论 文集中提取数据集，它包含 1990-2018 年 IEEEVis

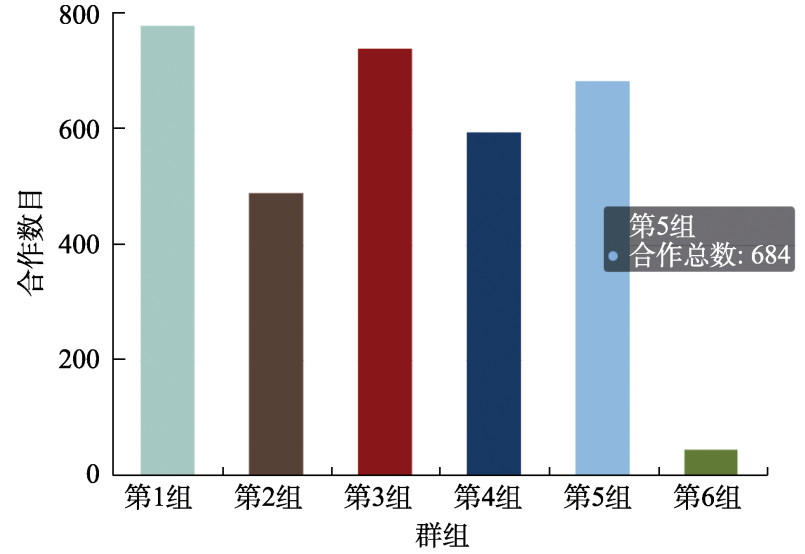

图 2 群组内部合作信息统计视图

可视化会议的所有论文, 共计 4340 条记录. 由于 论文数目有限, 结合 DBLP 数据集, 查找上述数据 集中的作者在除可视化外其他领域的论文信息， 最终根据作者间合作紧密程度对数据集中的作者 进行分组, 篮选群组合作的作者, 根据群组合作信 息展示学术研究人员协作网络中群组的演变模式. 本文使用 Java 和 MySQL 进行基本数据的篮选和处 理，探究群组协作随时间的演变模式. 结合第 2.2 节中提出的任务分类需求, 通过设计可视化元素 隐喻, 基于 JavaScript 库中的 Echarts.js ${ }^{1}$ 设计实现 可视化视图, 最终得到了研究人员的群组协作网 络视图.

(2) 视图分析. 通过将学术协作网络数据集映 射到本文提出的可视化元素上, 可得到学术协作 网络可视化视图, 如图 3 所示.

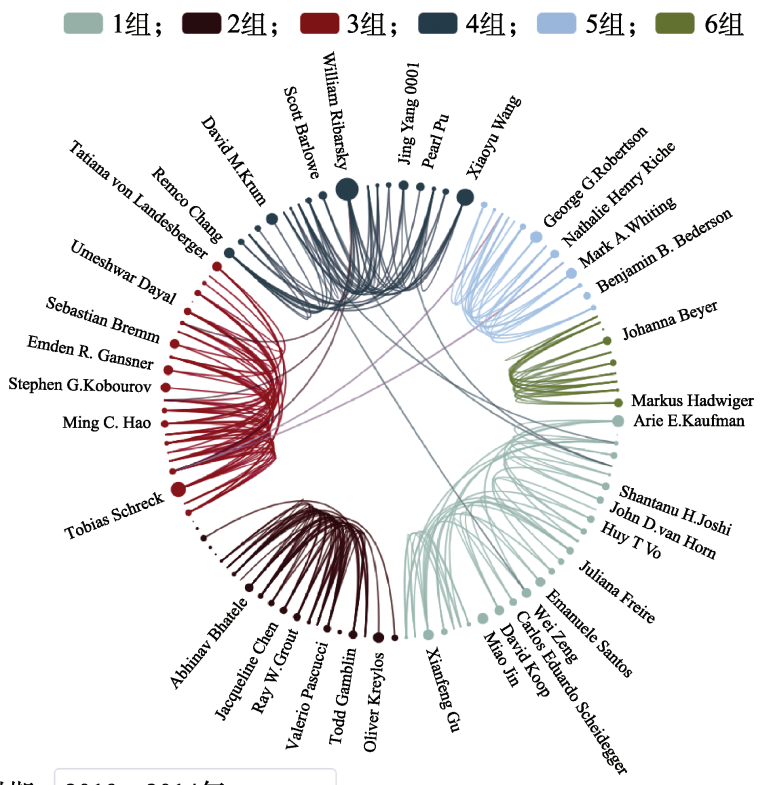

时期：2010-2014年

图 3 群组协作网络可视化

(1) https://echarts.baidu.com/index.html 
取数据集中的 6 组作者探究他们之间的协作 关系, 其中, 对学术作者群组的划分, 认定合作数 目超过一定程度的为同一组(本文中阈值设定为 6), 即为同一个研究领域的学术作者. 视图中不同颜 色的节点代表不同组的学术作者, 连线代表作者 之间协同合作论文，添加时间下拉框，可选择查看 不同时间段的作者合作情况. 从图 3 中可以看出， 大多数的合作关系都在群组内部，通常在学术研 究领域作者更容易和其研究领域内的人合作论文,
但也存在一些涉及其他领域的论文合作，从时间 的推进查看合作情况的演变, 也可以看到作者合 作领域的改变, 以预知其研究兴趣的转移.

图 4 中显示视图为本文设计的选择过滤的交 互手段. 如图 4a 所示为选择查看第 3 组学术作者 之间的合作概况，图 $4 \mathrm{~b}$ 所示为查看第 3 组和第 4 组学术作者以及 2 组之间作者的合作概况; 用户也 可以通过点击按钮查看更多作者之间的群组协作网 络可视化视图，以探索群组之间的协作关系.

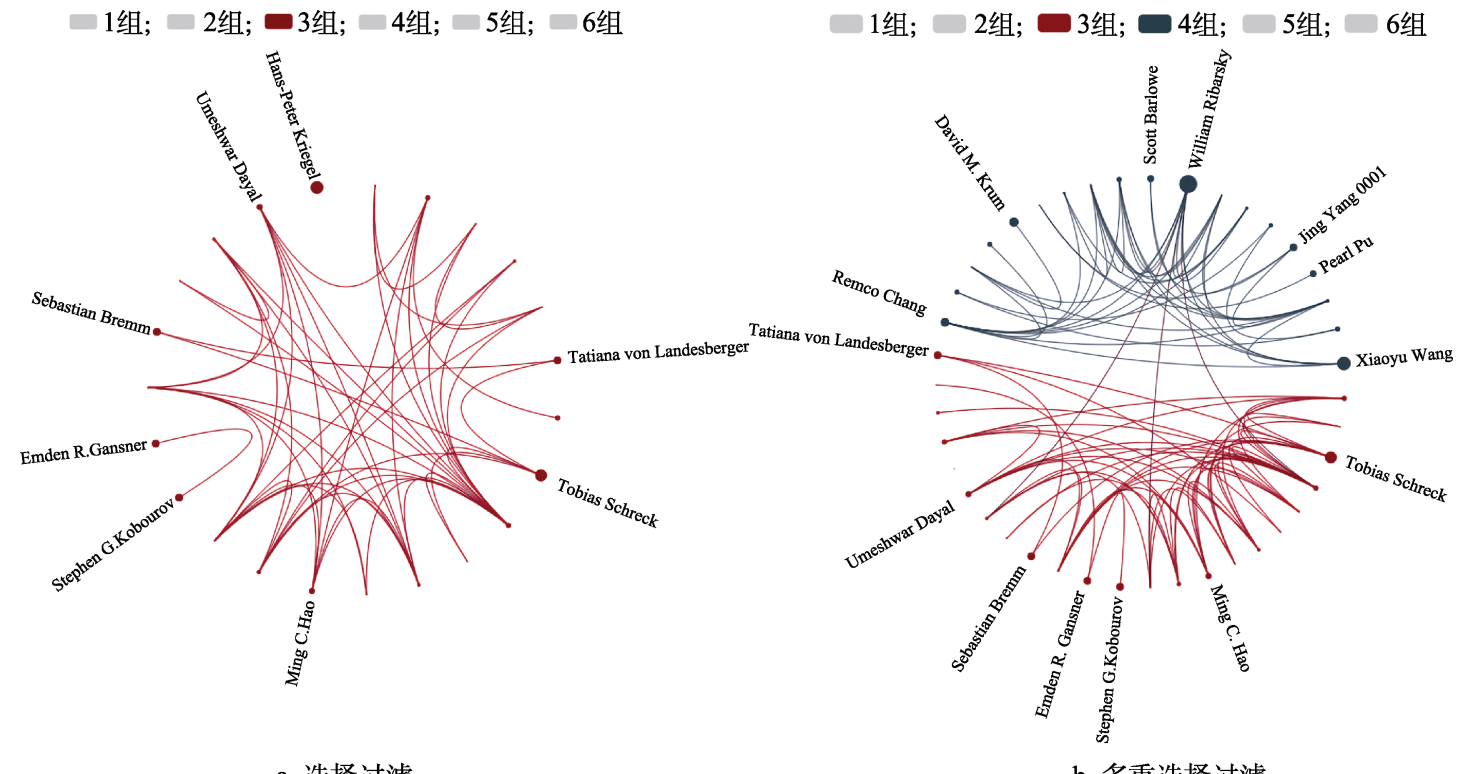

a. 选择过滤

b. 多重选择过滤

图 4 选择过滤的交互手段

为探究随时间推进群组协作关系的演变, 本 文设计添加时间表达，如图 5 所示，可查看 6 个群 组内部以及群组之间的网络协作关系的演化. 从 图 5 可以看出, 随时间演变, 群组内部作者及合作 情况更加紧密, 群组之间的合作状况也出现较多. 为方便查看特定群组之间协作关系，选择展示第 3 组和第 4 组作者协作网络可视化视图, 如图 6 所示, 从图 6a 可以看出, 群组数作者数量和发表合作论 文数量都相对较少, 2 个群组代表的节点之间没有 链接，表明 2 个群组在这个时间段之间没有合作关 系. 从图 $6 \mathrm{~b}$ 可以看出, 随着研究方向的深人, 2 个 群组内部的作者数目及合作论文数目也愈来愈多, 并且随着研究领域的扩展，它们之间也产生了节 点的链接，代表了作者之间跨领域的合作关系. 通 过选择不同时间段内的不同群组，可以查看其对应 的群组协作网络可视化视图, 从而发现群组协作关系 的演变, 探究跨领域的学者的网络协作, 以预知其研 究方向的转移.

\section{2 用户评估}

为了验证本文根据设置的群组协作任务建立 的抽象模型以及设计的可视化视图的有效性, 本 节通过用户评估实验进行验证。

\subsection{1 参与者}

邀请了 18 名计算机视觉专业的参与者 $(6$ 名女 性, 12 名男性), 年龄为 21 27 岁, 所有人的视力都 是正常的或矫正为正常的，没有任何色觉障碍. 参 与者包括本科生和研究生, 12 人熟悉或理解信息 可视化, 8 人熟悉或理解网络可视化.

\section{2 .2 评估任务及调查问卷}

为了使用户更加直接地了解可视化视图的 设计理念，根据第 2.2 节的组级任务分类，设置 一些用户在观察视图时需要完成的任务, 如表 2 所示.

用户完成任务后，对可视化视图有了一定深 人的了解, 之后需填写对本系统的调查问卷评分, 具体的问题如表 3 所示. 


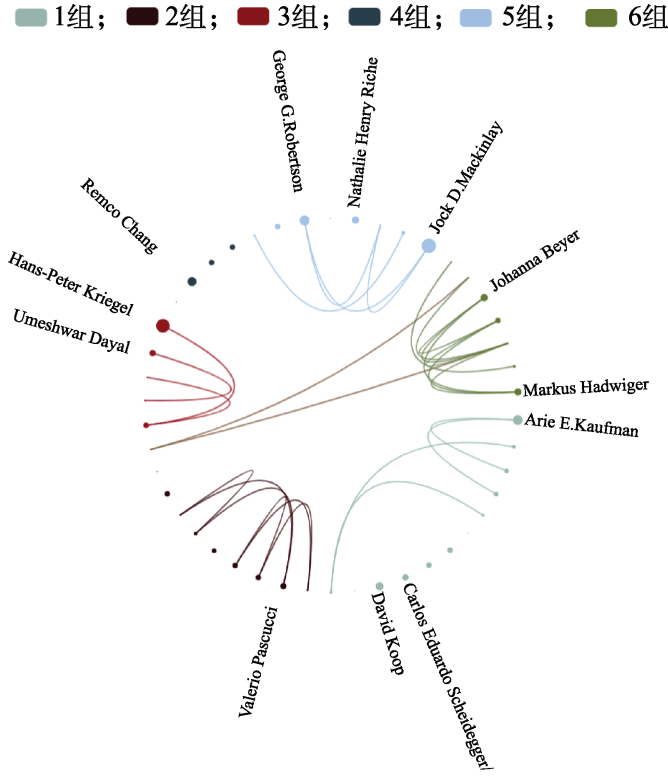

a. 1990-1994年间群组协作网络
1组; 2组; 3组; 4组; 5组; 6组

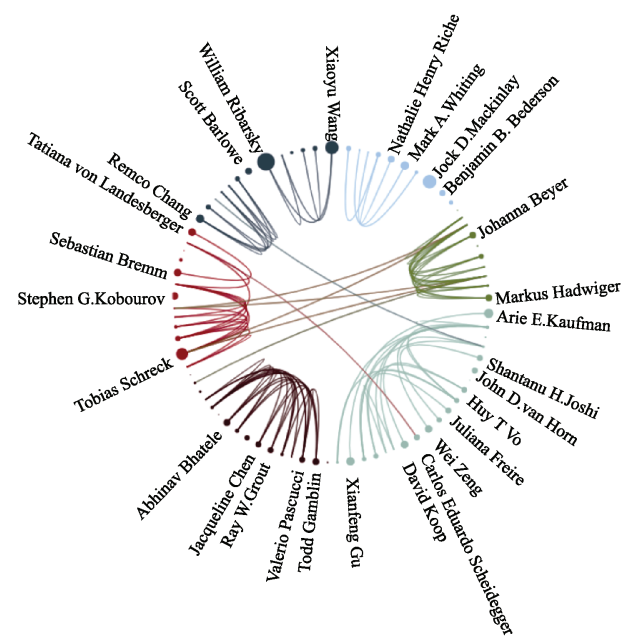

b. 2014-2018年间群组协作网络

图 5 多个群组协作网络演化视图

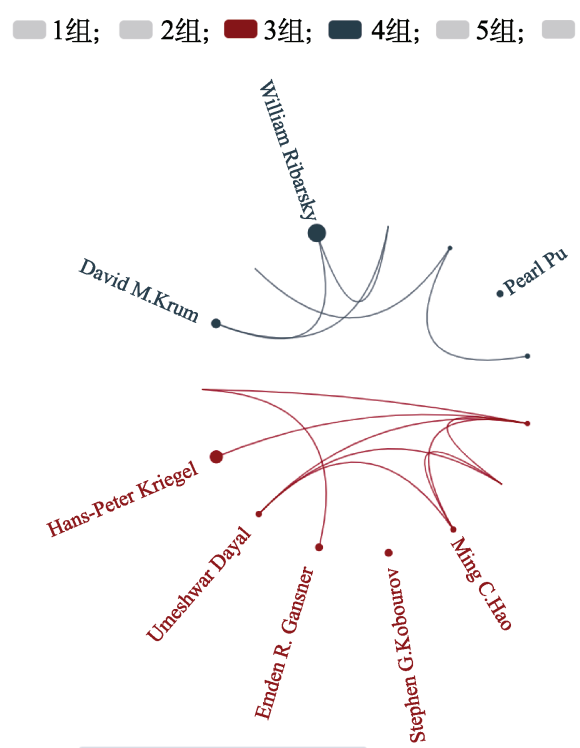

时期: 1998-2002年

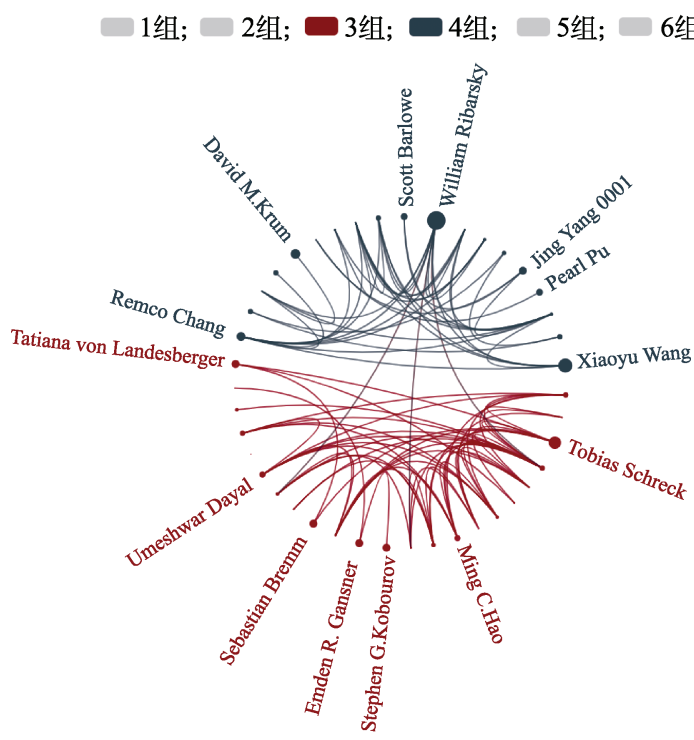

时期： 2010-2014年

a. 1998-2002年间 2 个群组协作网络

b. 2010-2014年间2个群组协作网络图

图 62 个群组协作网络演化视图

表 2 用户研究任务

\begin{tabular}{llc}
\hline 序号 & \multicolumn{1}{c}{ 任务 } & 任务分类 \\
\hline 任务 1 & $\begin{array}{l}\text { 查找 2002-2006 年间群组内部作者数目 } \\
\text { 最多的组 }\end{array}$ & $\mathrm{T} 1$ \\
任务 2 & 查找包含作者 Huamin Qu 的群组 & $\mathrm{T} 1$ \\
任务 3 & $\begin{array}{l}\text { 查找 2010-2014 年间组内合作数目最多 } \\
\text { 的组 }\end{array}$ & $\mathrm{T} 2$ \\
任务 4 & 查找与作者 Huamin Qu 有合作的所有组 & $\mathrm{T} 2$ \\
任务 5 & $\begin{array}{l}\text { 查找合作数目最多的 2 个组 } \\
\text { 任务 } 6\end{array}$ & 查看第3组与第 4组作者之间的合作情况 \\
\hline
\end{tabular}

表 3 调查问卷

\begin{tabular}{cl}
\hline 序号 & \multicolumn{1}{c}{ 问题 } \\
\hline 问题 1 & 可视化视图很容易学习 \\
问题 2 & 可视化设计与内容符合 \\
问题 3 & 可视化视图很容易发现群组 \\
问题 4 & 可视化视图容易发现群组之间的协作关系 \\
问题 5 & 可视化视图容易发现群组内部的协作关系 \\
问题 6 & 可视化视图方便观察群组协作关系的演变 \\
\hline
\end{tabular}

\subsection{3 实验过程}

在开始评估实验前, 参与者会进行相关背景 
介绍，使用户了解该项研究的大概内容，并可针对 提出的疑问进行答复，也设置一些问题让用户来 探索视图进行回答, 以确保用户对系统元素及功 能有更加深刻的了解. 然后要求参与者完成表 2 中 给出的任务, 待任务完成后填写表 3 中的调查问卷, 并和他们进行沟通, 总结他们对可视化视图的看法 以及相关建议. 问卷采用李克特 7 分制量表对测试 问卷进行分析，其中 1 分代表非常不同意, 7 分代表 非常同意，每一个参与者的实验大概持续 $30 \mathrm{~min}$.

\subsection{4 结 果}

实验结束后，收集所有参与者的调查问卷，对 问卷分数进行统计，对各个问题的统计平均分结 果如图 7 所示. 通过对统计数据进行分析发现, 参 与者都认为本文可视化视图很容易学习和与本文 研究内容相符合, 在群组协作关系的可视化方面 也表现较好. 在 6 个问题的评级中，问题 6 的方差 较大，通过查看调查问卷，与参与者进行进一步的 沟通, 发现 2 位参与者在探究群组协作关系的演变 时对视图存在一些疑问, 在网络规模较大时, 群组 之间的协作会更加复杂，节点之间的线条也会增 加, 可能会导致视觉混乱, 无法进一步探索群组协 作的演变规律.

本文针对提出的群组协作的组级任务设计可 视化视图进行群组协作的分析, 添加选择过滤的 交互方式可协助用户查看部分群组之间的协作情 况, 以减少视觉混乱; 但是, 在满足较大规模网络 关系的探索中确有一些不足之处, 之后也会进一 步完善和改进可视化视图, 以满足更大规模网络 关系的分析和探索.

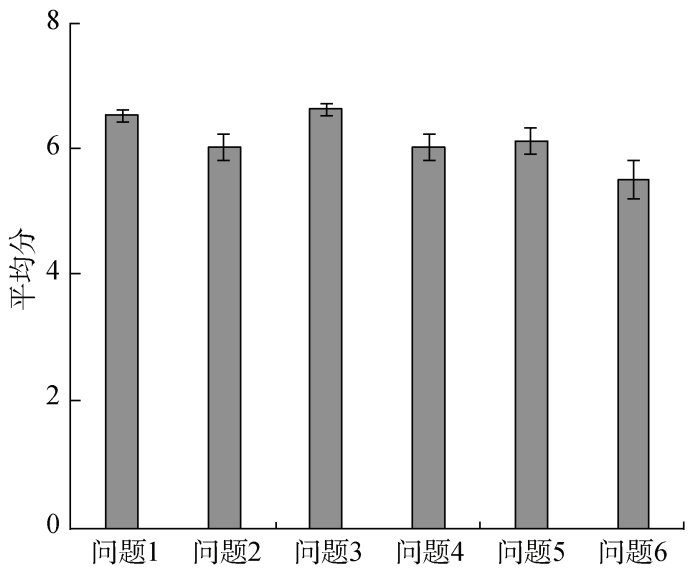

图 7 调查问卷的平均分

\section{5 结 语}

本文为分析群组协作社交网络关系中群组内
部以及群组之间的协作方式，首先对群组协作网 络探索中需要完成的任务进行分析, 归纳总结用 于图可视化的任务分类, 并提出群组级别任务分 类法, 定义一组新的任务来评估群组级别可视化 效果, 根据组级任务分类需求设计可视化视图, 展 示群组内部以及群组之间网络节点以及节点链接 之间的关系, 并且添加时间表达, 可供用户探索随 时间推进网络结构的演化模式. 最后, 在学术协作 文献数据集上进行具体案例分析, 并设置用户评 估，根据组级任务分类设置用户需要完成的任务， 收集用户对可视化视图评估的调查问卷, 通过最 终评估结果验证了本文设计可视化视图的有效性 和实用性.

本文也存在一些局限性，目前，用于分组的连 接关系数量阈值是固定的. 但是社交网络结构是 随时间变化的，一个固定的阈值可能不适用于所 有时间段. 另外，任务分组中，只考虑了单一时间 节点的群组关联关系也有一定的限制, 支持群组 协作关系分析的任务分类法一般包含 3 个维度： when, what, where ${ }^{[26]}$, 不同时间节点、不同群组实 体以及不同场景中的群组协作关系演变规律不同, 在之后的研究中, 将考虑在设置任务分类时, 从多 个角度考虑设置任务, 更深人探究群组协作关系 随时间的演化模式.

\section{参考文献(References):}

[1] Akhtar N, Javed H, Sengar G. Analysis of Facebook social network[C] //Proceedings of the 5th International Conference and Computational Intelligence and Communication Networks. Los Alamitos: IEEE Computer Society Press, 2013: 451-454

[2] Beck F, Burch M, Diehl S, et al. A taxonomy and survey of dynamic graph visualization[J]. Computer Graphics Forum, 2017, 36(1): 133-159

[3] Liu Zhen, Wu Xiangyang, Zheng Qiuhua. A survey of dynamic network visualization and visual analysis[J]. Journal of Computer-Aided Design \& Computer Graphics, 2016, 28(5): 693-701(in Chinese)

(刘真, 吴向阳, 郑秋华. 动态网络可视化与可视分析综述 [J]. 计算机辅助设计与图形学学报, 2016, 28(5): 693-701)

[4] Eades P, Huang M L. Navigating clustered graphs using force-directed methods[J]. Journal of Graph Algorithms and Applications, 2000, 4(3): 157-181

[5] Bach B, Pietriga E, Fekete J D. GraphDiaries: animated transitions and temporal navigation for dynamic networks[J]. IEEE Transactions on Visualization and Computer Graphics, 2014, 20(5): 740-754

[6] Rufiange S, Mcguffin M J. DiffAni: visualizing dynamic graphs with a hybrid of difference maps and animation[J]. IEEE Transactions on Visualization and Computer Graphics, 
2013, 19(12): 2556-2565

[7] Beck F, Burch M, Diehl S, et al. The state of the art in visualizing dynamic graphs[C]//Proceedings of the Eurographics Conference on Visualization. Aire-la-Ville: Eurographics Association Press, 2014: 83-103

[8] Arendt D L, Blaha L M. SVEN: informative visual representation of complex dynamic structure[EB/OL]. [2020-06-09]. https://arxiv.org/pdf/1412.6706.pdf

[9] He Q, Zhu M, Xie Z, et al. Revoler: visual analysis of relation evolutions in temporal data[J]. Journal of Computational Information System, 2015: 6555-6569

[10] Ogawa M, Ma K L. Software evolution storylines[C] // Proceedings of the ACM Symposium on Software Visualization. New York: ACM Press, 2010: 35-42

[11] Tanahashi Y, Hsueh C H, Ma K L. An efficient framework for generating storyline visualizations from streaming data[J]. IEEE Transactions on Visualization and Computer Graphics, 2015, 21(6): 730-742

[12] Bryan C, Ma K L, Woodring J. Temporal summary images: an approach to narrative visualization via interactive annotation generation and placement[J]. IEEE Transactions on Visualization and Computer Graphics, 2017, 23(1): 511-520

[13] Lu Q, Chai B J, Zhang H B. Storytelling by the StoryCake visualization[J]. The Visual Computer, 2017, 33(10): 1241-1252

[14] Beck F, Burch M, Vehlow C, et al. Rapid serial visual presentation in dynamic graph visualization[C] //Proceedings of the IEEE Symposium on Visual Languages and Human-centric Computing. Los Alamitos: IEEE Computer Society Press, 2012: 185-192

[15] Lu Q, Zhu X Y, Liu L, et al. An effective demonstration for group collaboration based on storyline visualization technology[C] //Proceedings of the 18th IEEE International Conference on Computer Supported Cooperative Work in Design. Los Alamitos: IEEE Computer Society Press, 2014: 47-52

[16] Sallaberry A, Muelder C, Ma K L. Clustering, visualizing, and navigating for large dynamic graphs[C] //Proceedings of the 20th International Symposium Graph Drawing. Heidelberg: Springer, 2012: 487-498
[17] Zhang Lisheng. The research and application of collaborative performance evaluation model for mobile collaborative groups[D]. Shijiazhuang: Hebei University of Economics and Business, 2012(in Chinese)

(张立生. 移动协同群组协作性能评估模型的研究与应用 [D]. 石家庄: 河北经贸大学, 2012)

[18] Palla G, Derényi I, Farkas I, et al. Uncovering the overlapping community structure of complex networks in nature and society[J]. Nature, 2005, 435(7043): 814-818

[19] Heer J, Boyd D. Vizster: visualizing online social networks [C] //Proceedings of the IEEE Symposium on Information Visualization. Los Alamitos: IEEE Computer Society Press, 2005: 33-40

[20] Brehmer M, Munzner T. A multi-level typology of abstract visualization tasks[J]. IEEE Transactions on Visualization and Computer Graphics, 2013, 19(12): 2376-2385

[21] Lee B, Plaisant C, Parr C S, et al. Task taxonomy for graph visualization[C] //Proceedings of the AVI Workshop on BEyond Time and Errors: Novel Evaluation Methods for Information Visualization. New York: ACM Press, 2006: 1-5

[22] Alper B, Riche N, Ramos G, et al. Design study of LineSets, a novel set visualization technique[J]. IEEE Transactions on Visualization and Computer Graphics, 2011, 17(12): 2259-2267

[23] Gansner E R, Hu Y F, Kobourov S. GMap: Visualizing graphs and clusters as maps[J]. IEEE Computer Graphics and Applications, 2010, 30(6): 54-66

[24] Collins C, Penn G, Carpendale S. Bubble Sets: revealing set relations with isocontours over existing visualizations[J]. IEEE Transactions on Visualization and Computer Graphics, 2009, 15(6): 1009-1016

[25] Tang J, Zhang J, Yao L M, et al. ArnetMiner: extraction and mining of academic social networks[C] //Proceedings of the 14th ACM SIGKDD International Conference on Knowledge Discovery and Data Mining. New York: ACM Press, 2008: 990-998

[26] Lu Q, Huang J, Zhang Q Y, et al. Evaluation on visualization methods of dynamic collaborative relationships for project management[J]. The Visual Computer, 2020, 130: 1-14 